\title{
Improving Spectral-Based Fault Localization Using Static Analysis
}

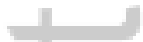

Neelofar*, Lee Naish, Jason Lee and Kotagiri Ramamohanarao

Department of Computing and Information Systems, University of Melbourne, Melbourne, Australia

$=$

\section{SUMMARY}

Debugging is crucial for producing reliable software. One of the effective bug localization techniques is Spectral-Based Fault Localization (SBFL). It helps to locate a buggy statement by applying an evaluation metric to program spectra and ranking program components on the basis of the score it computes. SBFL is an example of dynamic analysis - an analysis of computer program that is performed by executing it with sufficient number of test cases. Static analysis on the other hand, is performed in a non-runtime environment. We introduce a weighting technique by combining these two kinds of program analysis. Static analysis is done to categorize program statements into different classes and giving them weights based on the likelihood of being buggy statement. Statements are finally ranked on the basis of the weights computed by statements' categorization (static analysis) and scores computed by Spectral-Based Fault Localization metrics (dynamic analysis). We evaluate the performance of our technique on Siemens Test Suite (STS) and Flex (having seeded bugs seeded by expert developers), Sed (having mixture of real and seeded bugs) and Space (having real bugs). In our evaluation, proposed weighting technique improves the performance of a wide variety of fault localization metrics up to $20 \%$ on single bug datasets and up to $42 \%$ on multibug datasets. Copyright (C) 2010 John Wiley \& Sons, Ltd.
\end{abstract}

Received .

KEY WORDS: spectral debugging, static analysis, dynamic analysis, fault localization metrics

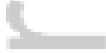

\section{INTRODUCTION}

Debugging software is an important and resource intensive task in software engineering, with $50 \%$ to $80 \%$ of software development and maintenance costs attributed to bug fixes [1]. Research suggests that of all debugging tasks, fault localization is the most time consuming and requires the most expertise [2]. Due to this cost, many researchers are studying and proposing effective approaches which involve automated tools to aid fault localization. There are many techniques proposed for fault localization and debugging in literature. Spectral-Based Fault Localization (SBFL) techniques [3][4][5][6] have gained high popularity in the last few years due to their simplicity. These methods extract program spectra-execution profiles of program components (statements, predicates, functions, etc.) and information on whether tests pass or fail. Using program spectra, fault localization metrics score program components, which are then ordered by the likelihood of containing the faults.

SBFL is not only of academic interest but has many practical applications in industry. A tool called Tarantula developed by the Spider Lab at University of Galifornia, uses the pass/fail statuses of test cases and the events that occurred during execution of each test case to offer the developer

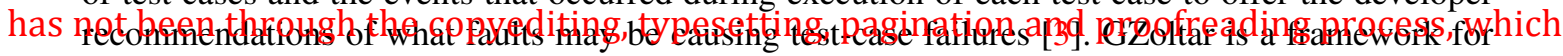
may lead to differences between this version and the Version of Record. Please cite this article

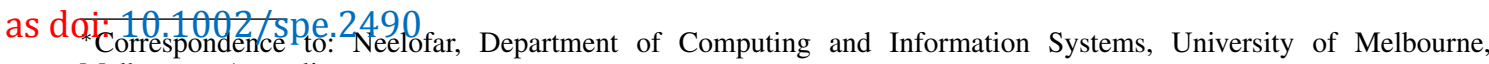
Melbourne, Australia 


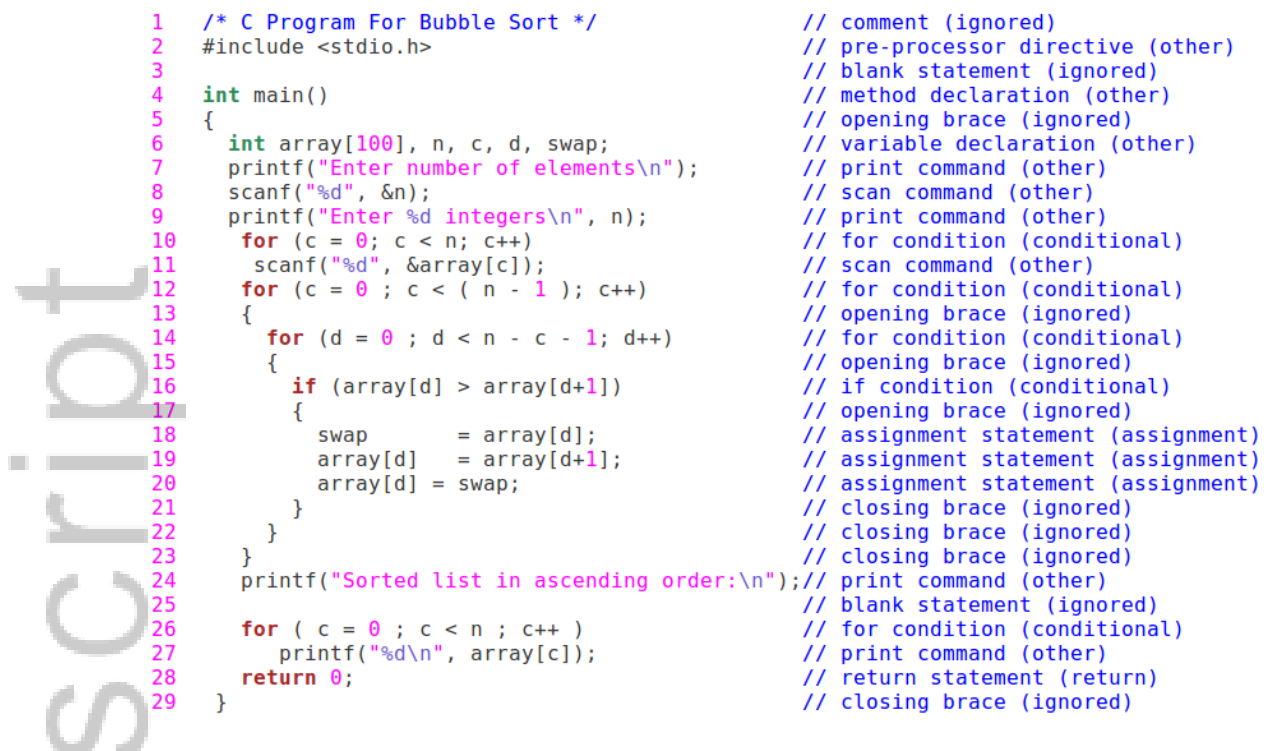

Figure 1. C Program Showing Different Categories of Statements.

automating the testing and debugging phases of the software development life-cycle. The framework is provided as an Eclipse plug-in and integrates seamlessly with JUnit tests [7]. The Cooperative Bug Isolation (CBI) system uses predicate spectra: predicates such as conditions of if-then-else statements are instrumented and data is gathered on whether control flow ever reaches that point and, if it does, whether the predicate is ever true [8]. In our study, we use spectral information of individual statements to localize bugs.

Spectral-Based Fault Localization uses only four parameters extracted during program execution: executed passed, not executed passed, executed failed and not executed failed. Although it has been shown to be effective, its fault localization accuracy is limited, since program syntax or semantics are not considered. Here we show that performance of these techniques can be improved by taking into account some additional information of program characteristics obtained using static analysis (see [9] for a survey of static analysis).

Studies show that some types of software bugs are more common than others. Common software constructs where it is more probable for software developers to introduce mistakes are in assignment (Null dereferencing, redundant assignment), out of range values in conditional statements (if constructs, switch, for, while and do-while loops), arithmetic exceptions (divide by zero, floating point exceptions) and buffer under/overflow [10]. Examples of some of these program constructs are shown in Figure 1. This research aims at improving the performance of SBFL techniques (dynamic analysis) by incorporating information gained by static analysis of program such as categorizing statements into different classes and estimating their likelihood of being buggy relative to other types of statements.

In this paper we make the following contributions:

- We use static analysis to categorize program statements into various classes. Static analysis can be used for extracting complex information about program like nesting levels, program structure and dependencies. In this study, however, we use simple static analysis to divide statements into different types or categories like assignment, return, control, function call etc.

- We investigate which category of program statements are more bug prone, based on which, these categories are given weights.

- Statements are ranked based on scores computed using fault localization metrics and weights computed based on statement's category. 


\begin{tabular}{|c|c|c|c|c|c|c|c|c|c|c|c|c|c|}
\hline & $T_{1}$ & $T_{2}$ & $T_{3}$ & $T_{4}$ & $T_{5}$ & $\overline{T_{6}}$ & $\overline{T_{7}}$ & $T_{8}$ & $T_{9}$ & $T_{10}$ & $e f$ & $e p$ & Score \\
\hline $\mathrm{S}_{1}$ & 1 & 1 & 1 & 1 & 1 & 1 & 1 & 1 & 1 & 1 & 3 & 7 & 0.5 \\
\hline $\mathrm{S}_{2}$ & 0 & 0 & 0 & 1 & 0 & 0 & 0 & 0 & 1 & 1 & 0 & 3 & 0.0 \\
\hline $\mathrm{S}_{3}$ & 1 & 0 & 0 & 0 & 1 & 0 & 0 & 0 & 0 & 1 & 1 & 2 & 0.3 \\
\hline $\mathrm{S}_{4}$ & 1 & 1 & 1 & 0 & 0 & 0 & 1 & 0 & 0 & 0 & 3 & 1 & 0.8 \\
\hline $\mathrm{S}_{5}$ & 0 & 1 & 0 & 0 & 0 & 1 & 1 & 0 & 1 & 0 & 1 & 3 & 0.2 \\
\hline \multicolumn{14}{|c|}{ I } \\
\hline $\operatorname{Res}$ & 1 & 1 & 1 & 0 & 0 & 0 & 0 & 0 & 0 & 0 & $F=3$ & $\bar{P}=$ & \\
\hline
\end{tabular}

Table I. Statement spectra with tests $T_{1} \ldots T_{10}$

- We evaluate our technique on benchmark programs written in $\mathrm{C}$ programming language. However, we expect that these results are equally applicable to other imperative programming languages as common types of bugs mentioned in [10] are not specific to a programming language.

- We perform our experiments on a range of programs including Siemens Test Suite, Space, Sed and Flex. Siemens Test Suite and Flex contains bugs seeded by expert developers, Sed have both real and seeded bugs while Space contains real software bugs.

The rest of the paper is structured as follows. Section 2 provides background information on SBFL, and gives the definitions of selected metrics from the literature that we use for comparison. Section 3 discusses our motivation behind this work, while we discuss our technique in detail in Section 4. Section 5 describes the experimental setup, details of the datasets we use in our experiments and our results. We list some threats to the validity of our results in Section 6. Section 7 briefly reviews some additional related work and Section 8 concludes.

\section{DEFINITIONS AND NOTATIONS}

SBFL methods use a set of tests, each classified as failed or passed; this can be represented as a binary vector, where 1 indicates failed and 0 indicates passed. For statement spectra $[5,6,11,12,13,14,15]$ which we use here, we gather data on whether each statement is executed or not for each test. This can be represented as a binary matrix with a row for each statement and a column for each test; 1 means executed and 0 means not executed. For each statement, four $a_{i j}$ values are ultimately produced, $i \in\{n, e\}$ and $j \in\{p, f\}$. These are the number of passed/failed test cases in which the statement was/wasn't executed - $\langle e f, e p, n f, n p\rangle$, where the first letter indicates whether the statement was executed $(e)$ or not $(n)$ and the second indicates whether the test passed $(p)$ or failed $(f)$. We use $F$ and $P$ to denote the total number of tests which fail and pass, respectively. Clearly, $n f=F-e f$ and $n p=P-e p$ and it is sometimes convenient to use $F, P$, ef (or $n f$ ) and $e p$ (or $n p$ ) rather than all four $i j$ values. Table I gives an example binary matrix of execution data and binary vector containing the test results. This data allows us to compute $F, P$ and $\langle e f, e p, n f, n p\rangle$ values.

Metrics, which are numeric functions, can be used to rank the statements. Most commonly, they are defined in terms of $e p$, ef, $n p$, and $n f$ values. Statements with the highest scores computed by the metrics are considered the most likely to be buggy. We would expect buggy statements to generally have relatively high $e f$ values and relatively low $e p$. Table II gives definitions of some of the well established metrics (scoring functions).

Programmers searching for a bug are expected to examine statements, starting from the highestranked statement, until a buggy statement is found. In reality, programmers may well modify the ranking due to other considerations, and checking correctness generally cannot be done by a single statement at a time. Evaluation of different ranking methods generally ignores such refinement and just depends on the score and in turn on rank order. Here we use a common measure, "rank percentage", which is the rank of the top-ranked buggy statement, expressed as a percentage of the 

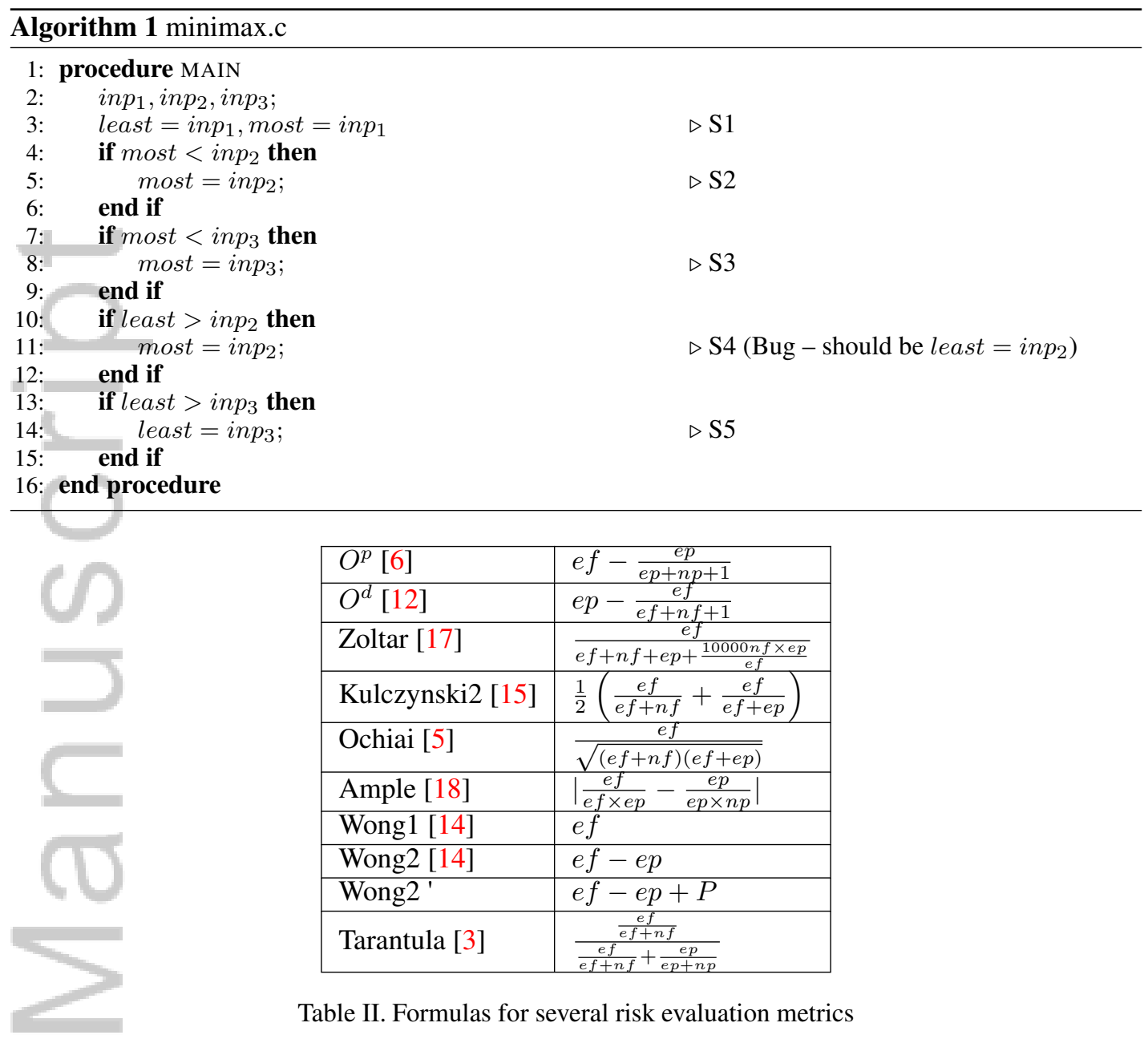

\begin{tabular}{|l|l|}
\hline$O^{p}[6]$ & $e f-\frac{e p}{e p+n p+1}$ \\
\hline$O^{d}[12]$ & $e p-\frac{e f}{e f+n f+1}$ \\
\hline Zoltar [17] & $\frac{e f}{e f+n f+e p+\frac{10000 n f \times e p}{e f}}$ \\
\hline Kulczynski2 [15] & $\frac{1}{2}\left(\frac{e f}{e f+n f}+\frac{e f}{e f+e p}\right)$ \\
\hline Ochiai [5] & $\frac{e f}{\sqrt{(e f+n f)(e f+e p)}}$ \\
\hline Ample [18] & $\left|\frac{e f}{e f \times e p}-\frac{e p}{e p \times n p}\right|$ \\
\hline Wong1 [14] & $e f$ \\
\hline Wong2 [14] & $e f-e p$ \\
\hline Wong2 & $e f-e p+P$ \\
\hline Tarantula [3] & $\frac{\frac{e f}{e f f n f}}{\frac{e f}{e f+n f}+\frac{e p}{e p+n p}}$ \\
\hline
\end{tabular}

Table II. Formulas for several risk evaluation metrics

program size. Results are averaged over all buggy programs in the benchmark set. Statements which are not executed in any test are ignored.

\subsection{Motivating Example}

In this section, we illustrate the steps involved in Spectral-Based Fault Localization using a simple example. Consider the faulty program minimax.c taken from [16]. The program fails due to the buggy statement $S 4$, where inp 2 should be assigned to least instead of most. To localize fault, coverage data from ten test cases $T_{1}$ to $T_{10}$ is collected. Seven of them pass and three fail. Their coverage details are given in Table I. For the purpose of simplicity, the spectral information of only few statements is considered. Ochiai [5] is applied to assign suspiciousness score to each statement (given in column score of Table I). The developer investigates the statements in descending order of their suspiciousness score until actual fault is located. Here Ochiai gives highest rank to the statement $S_{4}$, thus identifies the fault within the most suspicious program entity.

\section{MOTIVATION BEHIND CURRENT STUDY}

Intuitively some kinds of statements are more likely to have bugs than others. Common examples are complex conditional and assignment statements, nested conditions, and operations on arrays etc. In an effort to find most common bugs in Siemens Test Suite and Space (most commonly used 
Figure 2. Percentage of different categories of buggy and non-buggy statements in Siemens Test Suite (a) and Space (b).

benchmark data in the domain of SBFL study), we analysed these two benchmarks - Siemens Test Suite is a set of programs and relevant test cases collected by Siemens Corporate Research for a study of the fault detection capabilities of control-flow and data-flow coverage criteria [19], while Space is an interpreter for an array definition language [20]. Analysis revealed that out of 115 buggy versions of Printtokens2, Replace, Totinfo, Tcas, and Schedule (programs in Siemens Test Suite); 102 bugs are in assignment statements, return statements and conditional statements (for loop, while loop, and if-else conditions) while only 13 programs are found to have bugs in other program constructs. Although the bugs introduced in Siemens Test Suite are seeded, they are seeded by the expert developers and thus reflect real bugs, which according to studies, are more likely to occur in assignments and complex conditions as compared to variable declaration and initialization, and commands such as goto, continue, break etc. [10]. Our analysis of Space data, having real software bugs, confirmed previous studies, revealing 28 out of 38 bugs in assignments and conditions. Figure 2 shows percentages of different categories of buggy and non-buggy statements in Siemens Test Suite (a) and Space (b). It can be seen that more than $85 \%$ of the buggy statements in Siemens Test Suite belong to assignment, condition or return statement, in contrast to non-buggy statements, less than $30 \%$ of which belong to these groups. Similarly in Space, more than $75 \%$ of buggy statements are assignments, conditions and return statements, while less than $25 \%$ non-buggy statements belong to this group. Thus the distribution of different categories of buggy statements is unlike that for non-buggy statements. That means a high percentage of bugs in assignments, conditional statements and return statements are not due to their frequent occurrence in over all code, but instead, due to the nature of these statements. These statistics inspired us to incorporate the knowledge of statements' categories with spectral data to find whether it improves the performance of SPFL.

\section{OUR TECHNIQUE}

This section explains our technique of using Statement Categorization for improving SBFL. Statements are categorized into different classes and weights are computed for each class. These weights are used with fault localization metrics to compute final scores of the statements. Details of different steps of our methodology are given below.

\subsection{Statement Categorization}

The definition of "statement" and the way we introduce categories is influenced by the tools we use to extract program spectra. We use Gcov (part of the gcc compiler suite) to generate execution information of the program statements in passing and failing test cases. Gcov counts each line of code as an individual statement. Consider an example of a simple for loop:

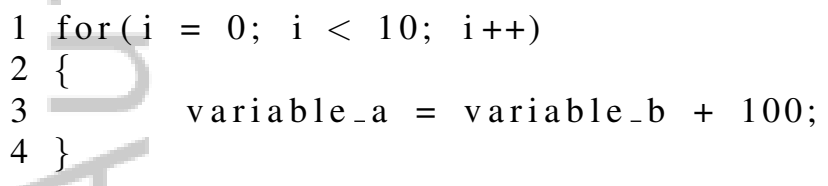

Line 1 of the for loop, having initialization, condition and increment would be considered as a single statement, while the opening and closing braces and assignment statement inside the body of for loop would be considered as three separate statements by Gcov. Similarly, Gcov counts blank lines and comments as statements as well. For example, the $\mathrm{C}$ program in figure 1 contains 29 statements in total, including blank statements $(3,25)$ and a comment (1).

We wrote a simple Python script to determine the category of $\mathrm{C}$ statements. If-then-else blocks, for loops, and while loops are grouped into a single category called conditional statements. Assignments and return statements are each given a separate category while all other types of statements such as 
function calls, variable declarations, goto, break etc. are grouped under the "other" category. Results shown in section 5.2 are based on using these 4 categories. However, other categories can be added to this list equally effectively. Many statements can be categorized using keywords such as return, if, else and while. Regular expressions are used to check more complicated cases such as assignment. Figure 1 shows various types of $\mathrm{C}$ statements and their categories.

We do not give weights to all the statements in our technique. In the example of a for loop above, statements 1 and 3 are categorized as condition and assignment respectively but the opening and closing braces are ignored. Similarly, although blank statements, comments and pre-processor directives are counted as individual statements by gcov, we ignore them while ranking as they are not executed and labelled them as "ignored" in figure 1.

In the experiments reported here, we do not use overlapping categories. Overlapping categories are where a single statement belongs to two or more categories. In the above for loop example, although the assignment statement at line 3 is inside the body of for loop, we do not consider it's category as conditional statement. Similarly, line 1 is not considered an assignment even though it contains an assignment $(i=0)$ within it. Likewise, conditions of if statements can have assignment operators embedded within them and assignment statements can have embedded conditional expressions, but our current script ignores the embedded expressions when determining the categories. Although we are not dealing with overlapping categories in this study, our technique is designed to accommodate them. Thus, refining the way statements are categorized, including the possibility of overlapping categories, is an obvious area of future research.

\subsection{Finding Weights}

Weights are computed for the statements after their categorization. A weight is computed for each category. The final score of a statement used in determing the ranking is defined by Equation 1 .

$$
\operatorname{score}(\operatorname{stm} t)=\text { score_metric }(\operatorname{stm} t) \times\left(1+\sum_{i=1}^{n} \delta_{i}(\operatorname{stm} t) \times w_{i}\right)
$$

In Equation 1, $i$ is the category of the statement, $n$ is the total number of categories we divided statements into, $\delta_{i}($ stmt $)$ is Kronecker delta function that returns value 1 if current statement belongs to category $i$ otherwise $0, w_{i}$ is the weight of the category $i$ and score_metric(stmt) is the score of a statement calculated by the chosen fault localization metric (based on the spectra for the statement). Scores of all the statements are calculated using Equation 1 and are ranked in descending order to find the most probable buggy statements.

Weights can be learnt using optimization methods such as simulated annealing, grid search or brute force search using training data. To simplify computing the weights, we limit the range of $w_{i}$ values. For the results presented here, we use the range [0 - 1]. Data is divided into training and testing partitions in a way that there is no mixing of the data between these two sets. The weights are learnt on training partition using optimization or fitness function that searches for the best weights within the defined range. By "Best Weights" we mean the weight values which give lowest rank percentage on training data for buggy statement(s). The effectiveness of the proposed method is determined by using these weights on testing data and determining rank percentage. The process of finding weights for each category of statement is explained using Algorithm 2. The function "FindBestWeightForStatements()" at line number 9 finds best weight values within the minimum and maximum weight range for different classes of statements while function "calcRankPercentage()" at line number 37 computes the average rank percentage using these weights. In order to avoid biased results, ten fold cross validation is used for learning and evaluation.

Table III shows weights computed for different categories of statements in various programs of STS and Space. The first column under each category of statement contains number of bugs of that category in training data, while second column shows weight computed for that category using our weighting technique. It can be seen that the value of weight assigned to a category increases with the proportion of bugs found in that category in training data. In Space for instance, the highest number 
Algorithm 2 Finding Weights for Different Classes of Statements

$\triangleright$ This algorithm shows weights computed for 4 categories of statements: control statements $\left(\alpha_{1}\right)$, assignment statements $\left(\alpha_{2}\right)$, return statements $\left(\alpha_{3}\right)$ and all other type of statements $\left(\alpha_{4}\right)$.

Input: Training Data

Output: Best weights for different types of statements $\left(\alpha_{1}, \alpha_{2}, \alpha_{3}, \alpha_{4}\right)$

: procedure MAIN(training_data)

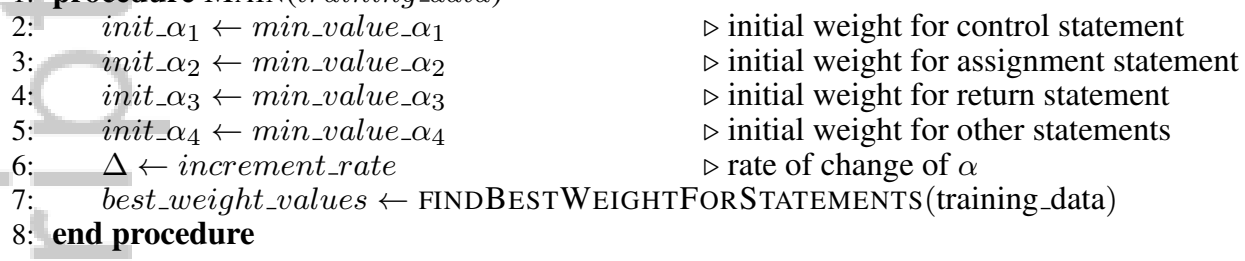

$\triangleright$ finds best weights for statements from a range of minimum and maximum weight values 9: procedure FINDBESTWEIGHTFORSTATEMENTS(training_data)

10: initial_rank_percentage $\leftarrow$ CALCRANKPER $\left(\right.$ init_ $\alpha_{1}$, init_ $\alpha_{2}$, init_ $\alpha_{3}$, init_ $\left.\alpha_{4}\right)$

11: $\quad$ best_ $\alpha_{1} \leftarrow$ init_ $\alpha_{1}$

12: best_ $\alpha_{2} \leftarrow$ init_ $\alpha_{2}$

13: $\quad$ best_ $\alpha_{3} \leftarrow$ init_o $\alpha_{3}$

14: $\quad$ best_ $\alpha_{4} \leftarrow$ init_ $\alpha_{4}$

15: $\quad$ while $\alpha_{1}<\max \_\alpha_{1}$ do

16: $\quad$ while $\alpha_{2}<\max \alpha_{2} \alpha_{2}$ do

17: $\quad$ while $\alpha_{3}<\max _{-} \alpha_{3}$ do

18: $\quad$ while $\alpha_{4}<\max _{-} \alpha_{4}$ do

19:

20:

21:

22:

23:

24:

25:

26:

27:

28:

29:

30:

31:

32:

33:

34:

35: $\quad$ return best_ $\alpha_{1}$, best_ $\alpha_{2}$, best_ $\alpha_{3}$, best_ $\alpha_{4}$

36: end procedure

$\triangleright$ calcula

procedure CALCRANKPER $\left(\alpha_{1}, \alpha_{2}, \alpha_{3}, \alpha_{4}\right)$

current_rank_percentage $\leftarrow$ CALCRANKPER $\left(\alpha_{1}, \alpha_{2}, \alpha_{3}, \alpha_{4}\right)$

if current_rank_percentage $<$ initial_rank_percentage then

initial_rank_percentage $\leftarrow$ current_rank_percentage

best_ $\alpha_{1} \leftarrow \alpha_{1}$

best_ $\alpha_{2} \leftarrow \alpha_{2}$

best_ $\alpha_{3} \leftarrow \alpha_{3}$

best_ $\alpha_{4} \leftarrow \alpha_{4}$

end if

$\alpha_{4}=\alpha_{4}+\Delta$

end while

$\alpha_{3}=\alpha_{3}+\Delta$

end while

$\alpha_{2}=\alpha_{2}+\Delta$

end while

$\alpha_{1}=\alpha_{1}+\Delta$

end while

38:

39:

40:

41:

42:

43:

44:

45:

46:

47:

48 :

49:

50:

for every spectral file do

for every statement in spectral data do determine statement category calculate statement score using fault localization metric multiply score with the weight according to the statement category assign score to the statement

end for

Rank statements in descending order of their scores

buggy_rank $\leftarrow$ Count rank of Buggy Statement

rank_percentage $\leftarrow \frac{\text { buggy_rank }}{\text { size of spectral data }} \times 100$

end for

return $r a n k \_$percentage

end procedure 


\begin{tabular}{|c|r|r|r|r|r|r|r|r|}
\hline Program & \multicolumn{2}{|c|}{ Conditional } & \multicolumn{2}{c|}{ Assignment } & \multicolumn{2}{|c|}{ Return } & \multicolumn{2}{|c|}{ Other } \\
\hline & bugs & weight & \multicolumn{1}{c|}{ bugs } & weight & bugs & weight & bugs & weight \\
\hline Space & 50 & 0.3 & 128 & 0.7 & 0 & 0.0 & 0 & 0.0 \\
\hline Replace (STS)* & 97 & 0.9 & 54 & 0.4 & 10 & 0.0 & 9 & 0.0 \\
\hline Schedule (STS) & 9 & 0.1 & 5 & 0.1 & 0 & 0.0 & 0 & 0.0 \\
\hline Print_Tokens2 (STS) & 16 & 0.4 & 0 & 0.0 & 3 & 0.1 & 1 & 0.0 \\
\hline
\end{tabular}

Table III. Weights computed for different categories of statements in various programs.

* Siemens Test Suite

of bugs are found in assignment statements and thus assignment category is given the highest weight of 0.7. Similarly for Replace, the highest weight is assigned to conditional statements as this is the most frequently occurring bug in this program.

\section{EXPERIMENTAL SETUP AND RESULTS}

\subsection{Experimental Setup}

5.1.1. Data Set We used the data for our experiments from Software-artifact Infrastructure Repositories (SIR)[21]. SIR is a repository of software-related artifacts meant to support experimentation with program analysis and software testing techniques. Datasets used in our experiments are the Siemens Test Suite, Flex, Sed and Space. Siemens Test Suite contains 7 programs in total i.e Printtokens, Printtokens2, Tcas, Replace, Totinfo, Schedule and Schedule2. These programs perform a variety of tasks: Tcas is an aircraft collision avoidance system, Schedule2 and Schedule are priority schedulers, Totinfo computes statistics given input data, Printtokens and Printtokens2 are lexical analyzers, and Replace performs pattern matching and substitution. For Printtokens2 and Schedule2, there are very few versions available in repository, most of which are either bugs introduced by deletion of a statement or commenting a statement. SBFL uses execution profiles of statements to localize bugs. As deleted or commented statements are not executed, there is no associated spectra. One way to evaluate a SBFL method for such programs is to replace the statement by an empty/non-operational statement, but due to very limited number of versions, we ignored these programs instead of editing and using them.

The Siemens Test Suite contains small programs having 563, 411, 173, 406 and 563 lines of code in Replace, Schedule, Tcas, Totinfo and Printtokens respectively. Space is a larger program having 9564 statements and functions as an interpreter for an array definition language (ADL). Flex is a lexical analyzer and consists of 10459 lines of code, while Sed is a Unix utility that parses and transforms text using a compact, simple programming language, having 14427 lines of code. Thus the Siemens Test Suite is an example of set of smaller sized programs while Flex, Sed and Space are large sized.

SIR contains single bug versions for all the programs. In order to generate two or multiple bug versions, we combined two or more single bug versions and tested the resulting programs against the provided test suites to generate spectra.

For learning purposes, we divided data into training and testing partitions, with $70 \%$ of data for training and $30 \%$ for testing. Data partitioning is done very carefully to fully avoid contamination of training data into testing data. Data contamination is very common especially in multi-bug benchmarks. To explain the reason behind the high probability of contamination, let us consider an example of generating two bug data from program versions 1,2 and 3. Three two bug versions generated by these three single bug versions are 1-2, 1-3 and 2-3. Although by using 1-2 and 1-3 for training, while 2-3 for testing, we are using separate two bug versions for training and testing, but still single bug versions 2 and 3 are present in both training and testing data. We avoid such contamination by generating two bug training and testing data from separate single bug nonintersecting subsets. As stated earlier, in order to avoid biased results, we generated ten different sets of training and testing data and computed all our results using ten fold cross validation. As 


\begin{tabular}{|c|r|r|r|r|}
\hline Statement & $\begin{array}{r}\text { +ve scoring } \\
\text { function } f\end{array}$ & $\begin{array}{r}\text { mixed scoring } \\
\text { function } g\end{array}$ & $f$ with weights & $g$ with weights \\
\hline 1(Category A) & 3 & 3 & 3.4 & 3.4 \\
\hline 2(Category B) & 3 & 3 & 3.1 & 3.1 \\
\hline 3(Category A) & 2 & -2 & 2.4 & -2.4 \\
\hline 4(Category B) & 2 & -2 & 2.1 & -2.1 \\
\hline
\end{tabular}

Table IV. An explanation of the poor performance of our technique with negative score generating metrics.

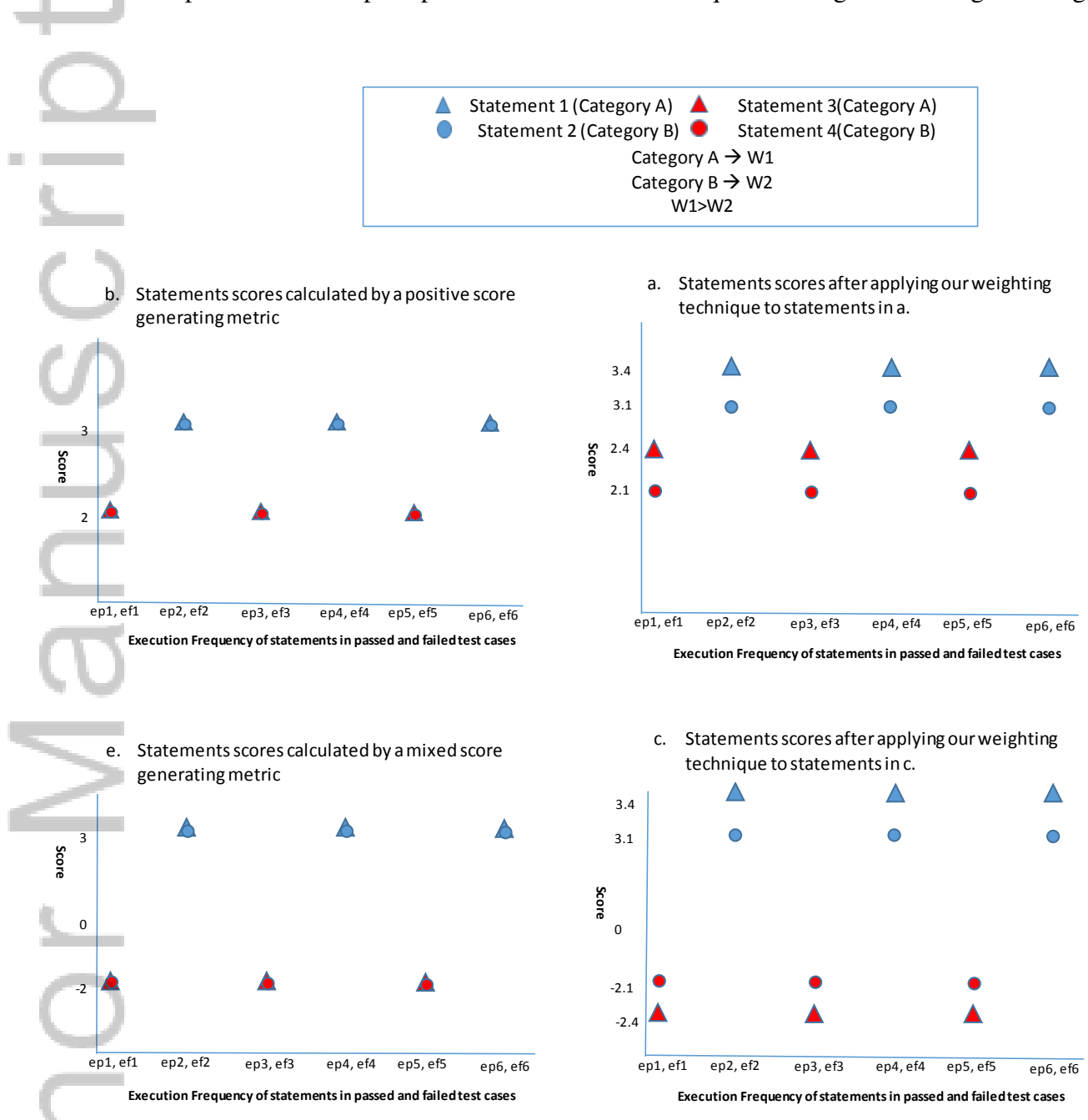

Figure 3. An explanation of the poor performance of our technique with negative score generating metrics.

learning of weights for different categories of statements is fixed and therefore does not have any hyper-parameter, no validation set is required for our experiments [22]. However, in our future work we wish to increase the number of categories of statements and the number of categories will be a hyper parameter requiring validation set to determine its effective value.

5.1.2. Range of Weights In our experiments, the range of the weights is set between 0 and 1 , with 0.1 increment rate. Effectively this range is between 1 and 2, as according to Equation 1, we add 1 to every weight to avoid the original score calculated by fault localization metric to be zero in case a statement is given zero weight. Although the range of weights used in this study is relatively small, a wider range could be used, particularly if data contains a large number of categories or overlapping categories. 
For very small range of weights, simple search methods like brute force do not take very long to execute, and results are more reliable due to exhaustive search. However, more sophisticated optimization methods like simulated annealing or genetic algorithms are required if the range of weights is very high or increment rate is very fine. The results shown in section 5.2 are based on weights computed using brute force search.

5.1.3. Fault Localization Metrics Used In our experiments we use a wide range of metrics and include metrics that have been shown to perform well for various benchmarks. $O^{p}[6]$ is at one extreme: it ranks statements primarily on their ef value and uses np to break ties when the ef values are equal. It was proved optimal for a single bug model program and this optimality result was strengthened in [11] to arbitrary single bug programs and test sets by restricting attention to strictly rational metrics, which are those whose value strictly increases in ef when ep is fixed and strictly decreases in ep (or increases in np) when ef is fixed. $O^{d}$ is at the other extreme: it ranks statements primarily on their np value and uses ef to break ties. In [12] it was shown to be optimal for programs with only deterministic bugs (which cause failure whenever they are executed). Zoltar is close to optimal for single bug programs and also performs well for multiple bug programs [15]. Kulczynski2 and Ochiai perform extremely well for the multiple-bug datasets of [15] which are also evaluated. A version of Ochiai also performed the best on benchmarks from the Software-artefact Infrastructure Repository (SIR) [23]. Tarantula was the first metric used for SBFL and though it performs relatively poorly for many datasets, a small adjustment makes it optimal for deterministic bugs [12]. Some other well known metrics like Wong1, Wong2 and Ample are also included in our evaluation to study their effectiveness in bug localization.

Our technique is designed to work with metrics which generate positive scores. Although most of the metrics in the field of spectral debugging are always positive, there are few such as Wong2 which generate mixed scores (positive and negative). However, these metrics can easily be converted to positive score generating metrics by adding a constant, without affecting original rank order of the scoring function. Wong2, for example, can be forced to generate positive score by adding $P$ (the total number of passed test cases) in its formula $(e f-e p+P)$. In [6] it is shown if $g(x)$ is monotonic in $x$, the rank order of function $f$ is preserved by the transformed function $g(f)$. Wong2' is thus the ranking equivalent of Wong2.

We now briefly explain why our technique does not work well with negative or mixed score metrics. Table IV shows 4 statements belonging to two different categories. Statements of category A are given weight $W 1$, while weight for category B statements is $W 2 ; W 1>W 2 . f$ and $g$ are two different metrics which compute positive and mixed scores respectively. Statements 1 and 2 are given the same score while 3 and 4 are given the same by $f$ and $g$. When we apply our weighting scheme-that computes final score of a statement by multiplying the score generated by fault localization metric with (1+weight) — column " $f$ with weights" shows $f$ gives higher score to statement 1 as compared to statement 2 as $W 1>W 2$. Similarly statement 3 is given more weight then statement 4 . However, mixed scoring metric gives lower weight to statement 3 as compared to statement 4 in spite of the fact that $W 1>W 2$. This can be further clarified by Figure 3 where blue triangles represent statement 1 (Category A), blue circles represent statement 2 (Category B), red triangles represent statement 3 (Category A), and red circles represent statement 4 (Category B). As $W 1>W 2$, triangles should always be given higher score after applying weights, however, Figure $3 \mathrm{~d}$ shows that red triangles are given lower final score as compared to red circles due to their negative scores. This limitation of our proposed technique can easily be resolved by transforming the mixed scoring function to positive scoring function as explained above.

\subsection{Experimental Evaluation}

This section shows evaluation results of our proposed technique for various single and multibug benchmark datasets. As per best of our knowledge, there is no real deterministic bug benchmark dataset available, we could not test our weighting technique on it. However, we strongly believe that the proposed technique will equally perform well on deterministic bug datasets. 
The evaluation results are given in tables V - IX. The tables give rank percentages of various metrics with and without applying our technique. Columns $1-10$ shows average rank percentage for ten fold cross validation while column Avg is the average of ten folds. Columns ConfidenceInterval and $p$-value gives 95\% Confidence Intervals and Wilcoxon signed-ranked test $p$-value for showing the statistical significance of our technique.

5.2.1. Single Bug Datasets We used single bug data from Siemens Test Suite, Flex and Sed. Space has very limited number of single bug versions available and thus can not be used for learning purposes. The evaluation results of our technique for single bug benchmarks are given in tables $\mathrm{V}$ and VI.

Table V shows average rank percentages of various metrics with and without applying our technique on single bug Siemens Test Suite. In column Avg, we can see that the performance of all the metrics has improved noticeably including $O^{p}$. Out-performing $O^{p}$ is significant because although there are countless possible metrics to choose from, it has been proved that for all programs and sets of tests, $O^{p}$ performs the best out of all "strictly rational" metrics [11].

Table V. Comparison of the average rank percentages of various metrics on single bug STS data with and without giving weights to the statements.

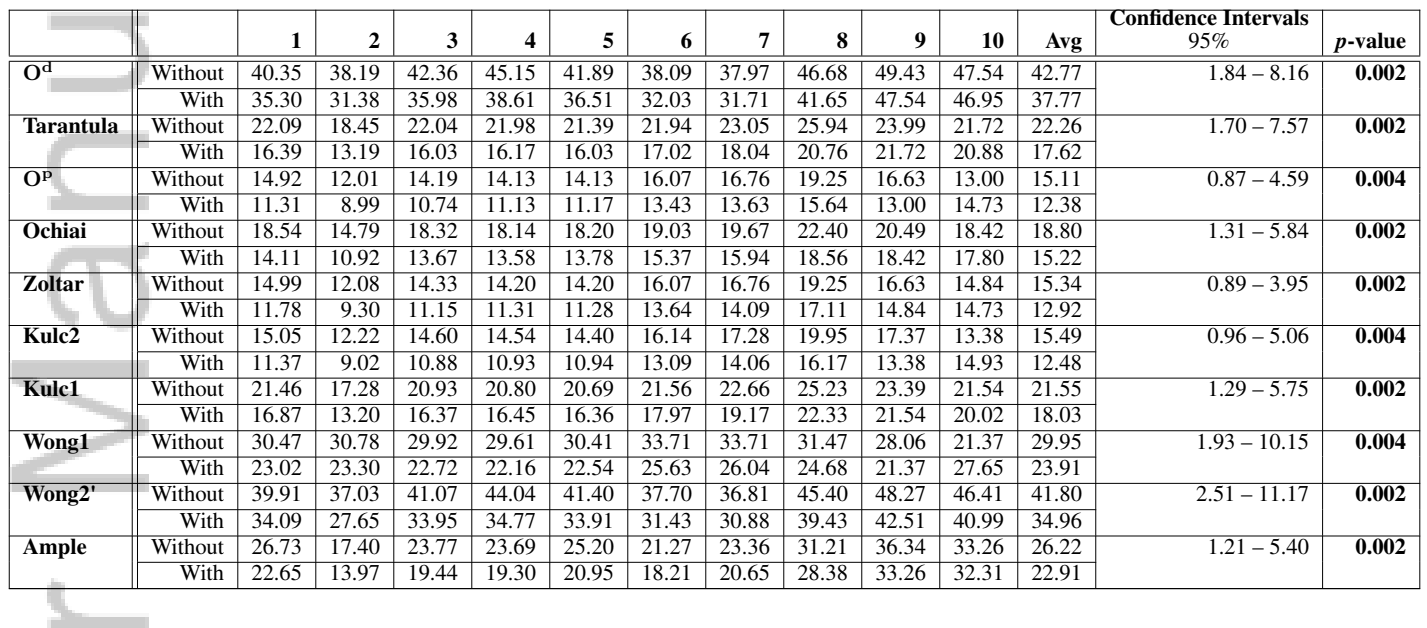

Table VI shows average rank percentages of various metrics with and without applying static analysis technique on single bug Flex and Sed datasets. We can see the performance improvement of many metrics in terms of decreasing rank percentage by using static information while ranking statements in order of their buggy probability. The performance of 6 out of 10 metrics significantly improved as shown in bold face in p-value column of the table. Notice that $O^{p}$ is not the best performing metric for this dataset, although we are labelling it as single bug. The reason behind less than the best performance of $O^{p}$ for this dataset is explained next.

We have generated data for Siemens Test Suite by taking program versions and test cases from SIR and made sure that only those program versions are included which satisfy Single Bug definition according to [6]. As per definition, if a bug is introduced by \#define in any program version, it will not be considered single bug if this \#define is used multiple times in the program. All the bugs in Flex and Sed data in Software Infrastructure repository are introduced by \#define and thus do not satisfy above mentioned single bug definition. Thus $O^{p}$ is not the best performing metric for this dataset.

In summary, apart from the discussion that which metric is performing best, we can see that our proposed technique improves the performance of all the metrics on Siemens Test Suite and most of the metrics on Flex and Sed data significantly. The performance improvement we have achieved here is because static information is used in addition to the program spectra. 
Table VI. Comparison of Average Rank Percentages of various metrics for single bug Flex and Sed

\begin{tabular}{|c|c|c|c|c|c|c|c|c|c|c|c|c|c|c|}
\hline & & 1 & 2 & 3 & 4 & 5 & 6 & 7 & 8 & 9 & 10 & Avg & $\begin{array}{c}\text { Confidence Intervals } \\
95 \%\end{array}$ & $p$-value \\
\hline \multirow[t]{2}{*}{$\mathrm{O}^{\mathrm{d}}$} & Without & 40.75 & 27.89 & 30.01 & 30.28 & 47.08 & 37.65 & 34.72 & 28.33 & 36.16 & 42.85 & 35.57 & \multirow[t]{2}{*}{$-3.19-0.76$} & \multirow[t]{2}{*}{0.232} \\
\hline & With & 39.46 & 29.22 & 35.53 & 35.30 & 50.39 & 39.41 & 37.54 & 27.59 & 33.56 & 39.78 & 36.78 & & \\
\hline Tarantula & Without & 23.36 & 16.12 & 17.50 & 12.57 & 23.52 & 16.46 & 19.23 & 12.40 & 18.65 & 22.37 & 18.22 & $0.83-4.40$ & 0.004 \\
\hline \multirow[t]{2}{*}{$\mathrm{O}^{\mathrm{P}}$} & Without & 29.96 & 23.84 & 19.10 & 20.76 & 22.33 & 20.32 & 25.87 & 27.06 & 35.07 & 33.74 & 25.81 & \multirow[t]{2}{*}{$-4.70-0.13$} & \multirow[t]{2}{*}{0.064} \\
\hline & With & 27.96 & 25.00 & 26.57 & 27.88 & 27.20 & 22.87 & 27.47 & 29.51 & 33.97 & 32.43 & 28.09 & & \\
\hline Ochiai & Without & 22.25 & 12.89 & 10.62 & 8.98 & 17.24 & 12.00 & 18.26 & 17.95 & 26.16 & 27.55 & 17.39 & $0.00-2.86$ & 0.049 \\
\hline Zoltar & With & 18.37 & 12.46 & 10.71 & 8.34 & 16.81 & 12.50 & 18.79 & 13.37 & 20.00 & 20.79 & 15.21 & $0.45-2.67$ & 0.006 \\
\hline \multirow[t]{2}{*}{ Kulc2 } & Without & 22.03 & 13.05 & 10.62 & 8.98 & 16.85 & 11.24 & 17.70 & 17.36 & 25.52 & 26.90 & 17.02 & \multirow[t]{2}{*}{$0.29-4.95$} & \multirow[t]{2}{*}{0.027} \\
\hline & With & 17.57 & 12.07 & 10.34 & 8.09 & 15.68 & 12.53 & 16.70 & 12.12 & 18.66 & 20.24 & 14.40 & & \\
\hline \multirow[t]{2}{*}{ Kulc1 } & Without & 22.72 & 13.20 & 10.68 & 8.98 & 18.19 & 13.32 & 19.83 & 18.75 & 26.80 & 28.29 & 18.07 & \multirow[t]{2}{*}{$0.77-3.41$} & \multirow[t]{2}{*}{0.002} \\
\hline & With & 19.63 & 12.05 & 8.90 & 6.74 & 15.51 & 12.99 & 19.18 & 16.48 & 23.76 & 24.53 & 15.98 & & \\
\hline Wong1 & Without & 28.61 & 28.90 & 25.64 & 28.27 & 25.86 & 27.29 & 30.17 & 32.83 & 34.25 & 31.21 & 29.30 & $0.64-3.82$ & 0.006 \\
\hline
\end{tabular}

5.2.2. Multi-bug Datasets We have used multi-bug datasets of Siemens Test Suite, Flex, Sed and Space for our experiments. As discussed earlier, multi-bug datasets are generated by combining two or more single bug versions of a program. In the experimental results we present here, we generated two bug versions of all the programs by combining two single bug versions.

Table VII shows average rank percentage of various metrics with and without applying our technique on multi-bug Siemens Test Suite. Results show that giving weights to statements on the basis of their categories results in quite a noticeable improvement in the performance of all the metrics, including Ochiai and Kulczynski2, which are considered as best performing metrics for multiple bug data [15].

Table VII. Comparison of the average rank percentages of various metrics on multi-bug STS data with and without giving weights to the statements.

\begin{tabular}{|c|c|c|c|c|c|c|c|c|c|c|c|c|c|c|}
\hline & & 1 & 2 & 3 & 4 & 5 & 6 & 7 & 8 & 9 & 10 & Avg & $\begin{array}{c}\text { Confidence Intervals } \\
95 \%\end{array}$ & $p$-value \\
\hline $\bar{O}^{\mathrm{d}}$ & Without & 35.22 & 39.51 & 37.58 & 43.27 & 40.84 & 40.50 & 38.41 & 38.61 & 34.34 & 31.90 & 38.02 & $2.25-10.00$ & $\overline{\overline{0.002}}$ \\
\hline \multirow{2}{*}{ Tarantula } & Without & 23.51 & 24.63 & 21.96 & 24.46 & 22.30 & 24.23 & 22.79 & 25.40 & 20.86 & 20.71 & 23.08 & \multirow[t]{2}{*}{$2.02-8.99$} & \multirow[t]{2}{*}{0.002} \\
\hline & With & 16.43 & 16.88 & 15.25 & 19.74 & 18.24 & 19.79 & 18.60 & 17.89 & 15.30 & 17.62 & 17.57 & & \\
\hline $\mathrm{O}^{\mathrm{P}}$ & Without & 27.19 & 24.98 & 22.99 & 22.73 & 24.26 & 23.35 & 24.42 & 29.29 & 26.54 & 29.10 & 25.48 & $1.30-5.76$ & 0.002 \\
\hline Ochiai & With & 16.26 & 16.58 & 16.80 & 19.82 & 19.52 & 18.80 & 17.65 & 16.64 & 13.42 & 18.89 & 17.44 & $1.51-6.70$ & 0.002 \\
\hline \multirow[t]{2}{*}{ Zoltar } & Without & 27.81 & 27.25 & 24.43 & 24.75 & 25.80 & 24.65 & 24.86 & 29.37 & 25.52 & 27.82 & 26.23 & \multirow[t]{2}{*}{$1.08-4.80$} & \multirow[t]{2}{*}{0.002} \\
\hline & With & 24.24 & 23.74 & 20.57 & 22.32 & 23.24 & 21.43 & 22.70 & 25.73 & 22.65 & 26.30 & 23.29 & & \\
\hline \multirow[t]{2}{*}{ Kulc2 } & Without & 21.35 & 21.05 & 18.96 & 18.87 & 20.39 & 20.45 & 21.26 & 24.47 & 19.22 & 26.15 & 21.22 & \multirow[t]{2}{*}{$0.01-3.75$} & \multirow[t]{2}{*}{0.049} \\
\hline & With & 17.99 & 18.66 & 18.22 & 19.62 & 20.43 & 20.68 & 20.99 & 18.43 & 15.74 & 22.61 & 19.34 & & \\
\hline \multirow[t]{2}{*}{ Kulc1 } & Without & 22.87 & 23.55 & 22.24 & 24.40 & 23.22 & 23.67 & 21.52 & 24.54 & 19.92 & 21.56 & 22.75 & \multirow[t]{2}{*}{$1.78-7.93$} & \multirow[t]{2}{*}{0.002} \\
\hline & With & 18.14 & 18.20 & 16.19 & 19.81 & 18.43 & 17.75 & 17.13 & 18.86 & 15.45 & 18.96 & 17.89 & & \\
\hline \multirow[t]{2}{*}{ Ample } & Without & 26.72 & 28.90 & 26.42 & 29.64 & 28.73 & 29.32 & 27.34 & 29.48 & 24.20 & 26.94 & 27.77 & \multirow[t]{2}{*}{$1.29-5.71$} & \multirow[t]{2}{*}{0.002} \\
\hline & With & 22.46 & 25.12 & 23.49 & 27.27 & 25.81 & 25.65 & 24.07 & 24.51 & 20.66 & 23.65 & 24.27 & & \\
\hline
\end{tabular}

Table VIII shows results by applying our technique on multi-bug Space data. Like multi-bug Siemens Test Suite, we can see a significant improvement in the performance of all the metrics by applying our weighting technique with Spectral-Based Fault Localization. Space is a large program having more than 9,000 lines of code, 13,000 test cases and have real software bugs. Thus improving metrics performance on Space data gives us confidence that our technique is equally applicable for real bugs which are random in nature in terms of their categories, unlike seeded bugs, which have limited categories depending on the developer who is introducing them.

Table IX shows rank percentages of various metrics with and without applying statement categorization technique on Flex and Sed data. As stated above, although we are using just four 
Table VIII. Comparison of the average rank percentages of various metrics on multi-bug Space data with and without giving weights to the statements. (Wong2' is the ranking equivalent of Wong2)

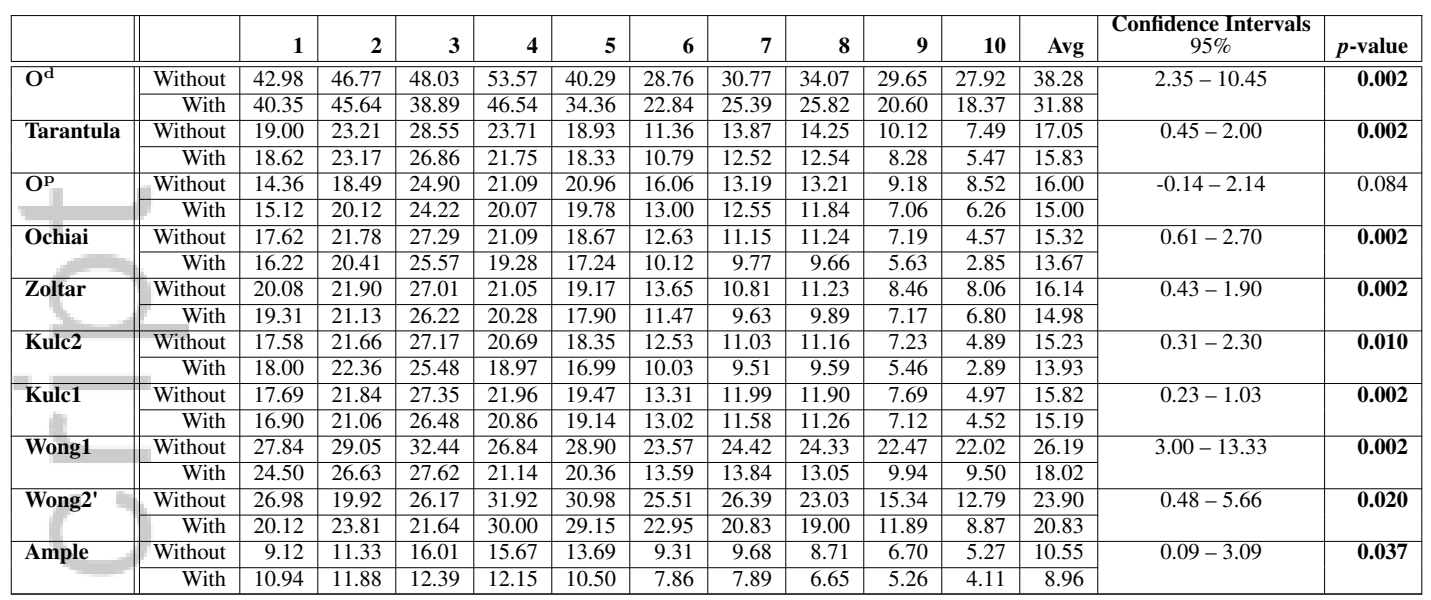

Table IX. Comparison of Average Rank Percentages of various metrics for multibug flex and sed

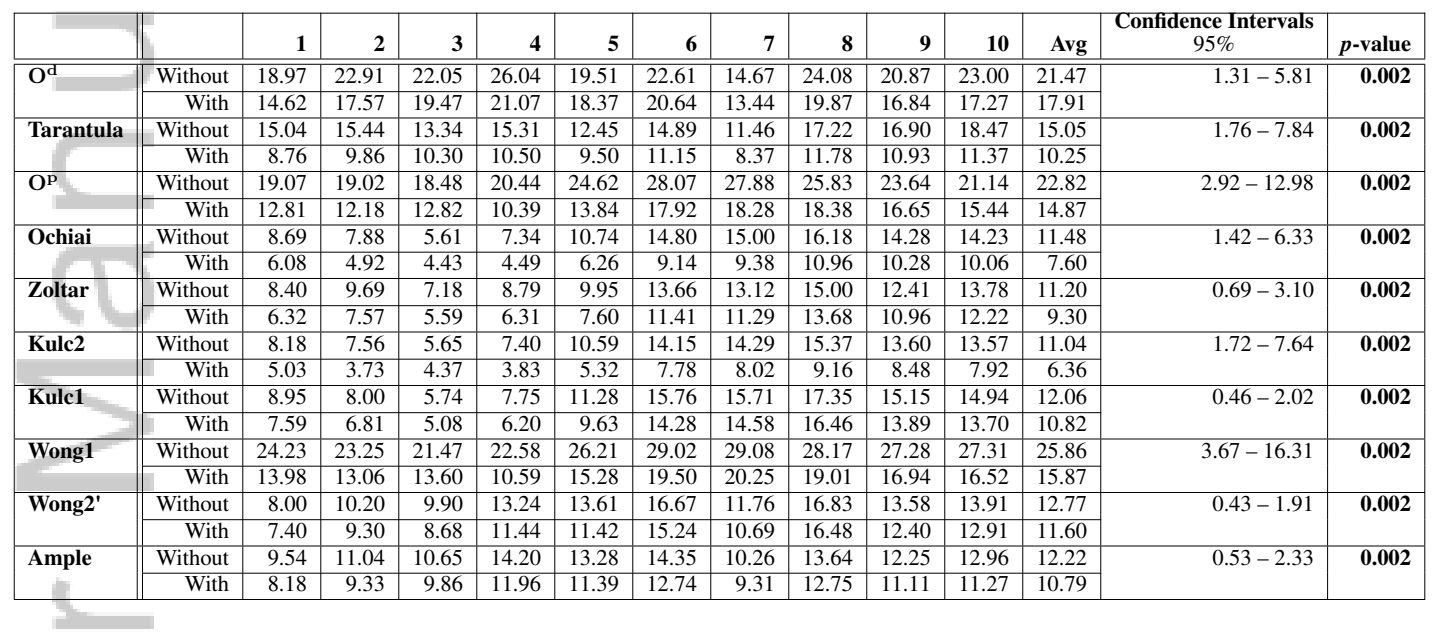

categories of statements for the results shown in Section 5.2, more categories can be added equally effectively. In order to test this, we added an extra category "function call" for the experiments on multi-bug Flex and Sed data. We can see that our technique still performs very well and improves the performance of all the metrics including $O^{p}, O^{d}$, Kulczynski2 and Ochiai. These results clearly show that using static analysis (statement categorization) with dynamic analysis (spectral debugging) significantly improves the performance of Spectral-Based Fault Localization.

\subsection{Performance Improvement In Terms Of Lines Of Code}

Rank percentage is the common measure of performance of fault localization metrics used in spectral fault localization literature. However, to emphasize the importance of our technique in real world debugging scenario, we calculate how much reduction in number of lines of code that a programmer has to investigate to locate the fault's site is achieved by applying our technique. Table X shows this information for multi-bug Space and Flex/Sed data. Column Average Rank Per With/Without shows average rank percentages of various metrics with and without applying our technique, while column \# of lines represents total number of lines a developer has to analyze to localize a fault. Column Difference shows how much reduction in terms of investigated lines of code is achieved by applying the proposed technique with spectral debugging. For Space, maximum difference is achieved by Wong1, for which developer has to observe 745 less lines of code to find 
Table X. Lines of Code a programmer has to examine to localize bugs with and without using our technique

\begin{tabular}{|c|c|c|c|c|c|c|c|c|c|c|}
\hline & \multicolumn{5}{|c|}{ Space } & \multicolumn{5}{|c|}{ Flex/Sed } \\
\hline & $\begin{array}{l}\text { Avg Rank Per } \\
\text { Without }\end{array}$ & \# of lines & $\begin{array}{c}\text { Avg Rank Per } \\
\text { With }\end{array}$ & \# of lines & Difference & $\begin{array}{l}\text { Avg Rank Per } \\
\text { Without }\end{array}$ & \# of lines & $\begin{array}{l}\text { Avg Rank Per } \\
\text { With }\end{array}$ & \# of lines & Difference \\
\hline $\mathrm{O}^{\mathrm{d}}$ & 38.28 & 3493.62 & 31.88 & 2909.20 & 584.42 & 21.47 & 2188.77 & 17.91 & 1826.24 & 362.53 \\
\hline Tarantula & 17.05 & 1555.82 & 15.83 & 1444.93 & 110.89 & 15.05 & 1534.53 & 10.25 & 1045.10 & 489.42 \\
\hline Ochiai & 15.32 & 1398.45 & 13.67 & 1247.95 & 150.50 & 11.48 & 1169.83 & 7.60 & 774.70 & 395.13 \\
\hline Zoltar & 6.14 & 1473.15 & 14.98 & 1366.88 & 106.27 & 11.20 & 1141.49 & 9.30 & 947.54 & 193.96 \\
\hline Kulc2 & 15.23 & 1389.81 & 13.93 & 1271.04 & 118.77 & 11.04 & 1125.07 & 6.36 & 648.83 & 476.24 \\
\hline Wong2 & 23.90 & 2181.36 & 20.83 & 1900.58 & 280.78 & 12.77 & 1301.81 & 11.60 & 1182.06 & 119.75 \\
\hline Ample & 10.55 & 962.69 & 8.96 & 818.00 & 144.69 & 12.22 & 1245.38 & 10.79 & 1099.97 & 145.41 \\
\hline
\end{tabular}

bug. This reduction in manual code analysis is significant for other metrics too. Similarly, for Flex /Sed, maximum performance improvement in terms of decrease in investigated lines of code by Wong1 is 1017 lines, which is a great improvement. Thus, a developer has to investigate much less number of lines of code to localize bugs by applying our statement categorization technique.

\subsection{Statistical Significance}

In order to find that the differences in the average rank percentage computed by applying our technique are significant as compared to without applying the weighting technique, we use Wilcoxon signed rank test at 5\% significance level [24]. We establish an alternative hypothesis; the bug localization performance using our proposed statement weighting approach improves as compared to using the unweighted approach. The null hypothesis is that there is no difference between the rank percentages computed by the two approaches. The $p$-value column in tables V, VI, VII, VIII and IX shows the $p$-value computed by Wilcoxon signed rank test on single and multi-bug data sets of the STS, Space, Flex and Sed. The $p$-value of 0.05 is a normal standard for statistical significance which is met for almost all the best performing metrics on the benchmarks we use in this research. The very small $p$-values mean we can be confident that our proposed technique of incorporating static information does increase performance for the metrics used in this research. To further validate the results, we include $95 \%$ confidence intervals showing the range of improvement in rank percentage of various metrics by applying our technique. In table VIII for instance, the range of improvement in rank percentage of Ochiai and Kulczynski2 is $0.6-2.7$ and $0.3-2.3$ respectively. For metrics like Wong 1 and Wong2, this range is even higher i.e. 3 - 13.3 for Wong1 and $0.4-5.6$ for Wong2. These results thus show that we are confident that our proposed method improves the performance of Spectral-Based Fault Localization metrics reasonably.

\section{THREATS TO VALIDITY}

Our proposed enhancement to SBFL relies on the assumption that statistical properties of training data and test data are very similar. We have used 10 fold cross validation in our evaluation to make sure the reported results are statistically significant which are shown by less than $0.05 p$-value for most of the metrics in tables V - IX. We have have used the same data sets that most published work in SBFL. However, in the following we will address the following issues:

We have chosen a wide spectrum of metrics for our experiments; best performing metric on single bug benchmarks $\left(O^{p}\right)$, deterministic bug benchmarks $\left(O^{d}\right)$, multiple bug benchmarks (Ochiai and Kulczynski2) and many others. Although we have not reported all SBFL functions (over 150 of them) we believe our results will hold good for any SBFL function as long as the statistical properties of training data and test data are very similar.

Results in section 5 are evaluated based on programs written in $\mathrm{C}$ programming language. Although common bugs mentioned in [10] are not specific to any programming language, there is a possibility that the proposed technique may not improve significantly on programs written in languages other than $\mathrm{C}$. 
Our experiments on several benchmarks with a wide range of fault localization metrics have consistently shown significant improvements in localizing faults with high confidence. Even the best performing metrics like $O^{p}$, Ochiai and Kulczynski2 have all uniformly improved their performance by using static analysis based weighting technique. However, unfortunately there are not many datasets available, and we have used only four publicly available benchmarks to validate our results; The Siemens Test Suite and Flex (having manually induced bugs by expert developers), Sed (having real and seeded bugs) and Space (having real software bugs). Our proposed technique performed significantly better using our enhanced method on Space and Sed having real bugs as on the Siemens Test Suite and Flex, but to further consolidate the claims, experiments on other datasets would be beneficial and we are endeavouring to collect such datasets.

\section{OTHER RELATED WORK}

Automated fault localization is an active research area and a huge literature is available showing different techniques proposed for this. A detailed survey of state of the art techniques in the field of software fault localization is provided in [25]. In this section we discuss some of the related studies.

Kai et al. proposed a tool called LOUPE. This automatic software fault localization tool gathers run time information and uses static analysis such as control and data dependencies analysis of the program. Based on this information, multiple spectra-specific models are built. Suspiciousness (ranking scores) of statement is produced based on the models. In our proposed approach, we gather additional information of categories of statements and compute the appropriate weights using machine learning [26].

Wanzhi introduced an initial proposal using program slicing technique and statistical method to build program slicing spectra before performing ranking of suspicious program statements. The statistical method extracts dependencies between program statements using software dependencies graphs (SDG) and refines execution history. There is no empirical result from their initial study [27]. In another related work, Hua et al. proposed a frequency weighting approach with program spectra where weighting is provided to the number of times each program statement executed by test cases. This approach shows statistically significant improvement of bug localization performance as compared to using binary information of test executions, especially on multiple bug programs. Our proposed approach uses weighting based on the different categories of program statements such as conditional, assignment and return statements [28].

Naish et al. uses probabilistic methods to improve the search strategy for declarative diagnosis of logic programming languages. Part of the algorithm used assigns a probability of being buggy to each clause of the program, based on (amongst other things) the total number of times each clause is used in the passed tests and a single failed test. Instead of probability, we are giving weights to the program statements based on the category they fit in [29].

The details of the few of the metrics we included in our research and some other techniques for SBFL are given below.

Most of the formulas or metrics used in SBFL are not designed specifically for software fault localization. Jaccard for example used for biological classification for the first time and Ochiai used in marine zoology [30]. Tarantula was the first metric designed for spectral fault localization [13]. Few other widely used metrics are Ample [18], Zoltar [17], Wong [14], $O^{p}[6]$ and $O^{d}$ [12] etc. $O^{p}$ and $O^{d}$ haved been mathematically proved to be the best "strictly rational" metrics for single and deterministic bug data respectively, they however, do not perform equally well on multiple bug data. A newly introduced class of metrics called "Hyperbolic Metrics" performs equally well on single and multiple bug data [31].

There are few techniques available in literature for multiple bug problem which are combination of Spectral-Based with machine learning or model based approaches. Some of these are given below.

James et al. present a clustering approach for debugging in parallel in presence of multiple bugs. Using fault localization information from program execution and behaviour models, they develop a technique that automatically partitions the failing test cases into clusters that target different faults. These clusters are called "fault focusing clusters". Each fault focusing cluster is then combined with 
all the passing test cases to get a specialized test suite that targets a single fault. These specialized test suits can then be assigned to different developers who can work in parallel for debugging and localizing bugs [32].

Abreu el al. present a multi fault localization technique called "BARINEL" by combining SBFL and model based reasoning. Model based approaches are more accurate as compared to spectral fault localization but due to their computational complexity they are very expensive for large applications. BARINEL, however, uses effective candidate selection process that reduces it's complexity and make it better candidate for large programs as well [33].

Wong et al. propose a crosstab-based statistical fault localization technique(CBT). The technique uses statement based coverage information. A comparison has been made between CBT and Tarantula and results prove CBT better then tarantula. The technique is claimed to be effectively applicable for multiple bug programs [34].

SBFL is one of many active research areas for fault localization. Some other techniques available in literature are:

Slicing and Dicing are considered as one of the oldest techniques in debugging and fault localization. Slicing refers to the piece of program code that affects the value of any variable while dicing is the part of program, which appears in one slice but not in another. These approaches narrow down the program part, which is more likely to be buggy so that developer can concentrate on a small part of the code for fault localization [15][35] [36][37].

Model based approaches compare the description of the correct behaviour of the system (model) to the observed behaviour. The difference between the actual model and observed behaviour may lead to model components that, when assumed to deviate from their normal behaviour, may explain the observed behaviour[38] [39]. A combination of model based techniques with other fault localization techniques have been proposed in literature [40][41][42][43].

Mutation based fault localization (MUSE) identifies the fault or bug by utilizing the information obtained by mutating the faulty and correct statement [44]. The technique uses the intuition that if a faulty statement is mutated, it will cause more tests to pass than average and if a correct statement is mutated, it will cause more tests to fail then average [45] [46][47]. [48][49] uses mutants spectra and shows that it localizes bugs more effectively than statement spectra even in the case where mutant sampling is used. More than $90 \%$ of the faults are localized with only $10 \%$ of code investigated.

State based approaches aim to localize the bugs by observing the changing state of the program and identifying the failure inducing circumstances [50][51][52]. Failure Inducing Circumstances refer to the input to the test cases which causes it to fail e.g. program input (particular URL input fails the web browser), User Interaction (keystroke of the user causing the program to fail) and changes to the program code.

There are many studies found that test reduction and test selection could help in improving the performance of fault localization technique [53]. Recently, many machine learning and data mining approaches are proposed in this area which can be used together with spectra-based approaches. Most of the work is done in test case clustering in this domain [32][54][55]. Le et al. propose a technique that combines spectral debugging and information retrieval to localize software bugs [56].

\section{CONCLUSION AND FUTURE WORK}

Spectral-Based Fault Localization uses a limited knowledge of execution of program components in passed and failed tests to localize bugs. We propose a technique that significantly improves the performance of fault localization metrics by incorporating information about categories of statements. The estimated likelihood of a statement being buggy depends both on its execution profile and a weight computed based on its category. We tested our technique on single and multiple bug benchmarks from the Siemens Test Suite, Space, Flex and Sed. Results show a major improvement in performance of all the fault localization metrics including the best performing metrics for single and multiple bug benchmarks. 
The improvements in performance are particularly encouraging because the static information we have incorporated so far is very simple. With more static information incorporated, we would expect even greater improvements in performance. It is a common observation that deeply nested, complex and large statements have a higher chance of being buggy. We plan to incorporate this information in learning weights to further improve our technique as future work. Furthermore, we are using only a small number of categories of statements in our experiments at this stage. We plan to statically analyze the programs more deeply to incorporate more classes and experiment with intersecting or mixed classes. The current study evaluates our technique using statement based spectra. We plan to use other types of spectral such as branch, data-flow, mutants etc. to further validate our findings.

\section{ACKNOWLEDGEMENTS}

We would like to thank the anonymous reviewers for their insightful comments on the earlier version of this manuscript, as their comments led us to an improvement in the presentation of this work.

\section{REFERENCES}

1. Collofello JS, Woodfield SN. Evaluating the effectiveness of reliability-assurance techniques. Journal of systems and software 1989; 9(3):191-195.

2. Vessey I. Expertise in debugging computer programs: A process analysis. International Journal of Man-Machine Studies 1985; 23(5):459-494.

3. Jones JA, Harrold MJ, Stasko J. Visualization of test information to assist fault localization. Proceedings of the 24th international conference on Software engineering, ACM, 2002; 467-477.

4. Liblit B. Cooperative Bug Isolation. PhD Thesis, University of California 2004.

5. Abreu R, Zoeteweij P, van Gemund A. An evaluation of similarity coefficients for software fault localization. PRDC'06 2006; :39-46.

6. Naish L, Lee HJ, Kotagiri R. A model for spectra-based software diagnosis. ACM Transactions on software engineering and methodology (TOSEM) August 2011; 20(3).

7. Campos J, Riboira A, Perez A, Abreu R. Gzoltar: an eclipse plug-in for testing and debugging. Proceedings of the 27th IEEE/ACM International Conference on Automated Software Engineering, ACM, 2012; 378-381.

8. Liblit B, Naik M, Zheng A, Aiken A, Jordan M. Scalable statistical bug isolation. Proceedings of the 2005 ACM SIGPLAN 2005; 40(6):15-26.

9. Wögerer W. A survey of static program analysis techniques. Technical Report, Citeseer 2005.

10. Vipindeep V, Jalote P. List of common bugs and programming practices to avoid them. Electronic, March 2005;

11. Naish L, Lee HJ, Kotagiri R. Spectral debugging: How much better can we do? 35th Australasian Computer Science Conference (ACSC 2012), CRPIT Vol. 122, CRPIT, 2012.

12. Naish L, Lee HJ. Duals in spectral fault localization. Proceedings of ASWEC 2013, IEEE Press, 2013.

13. Jones J, Harrold M. Empirical evaluation of the Tarantula automatic fault-localization technique. Proceedings of the 20th ASE 2005; :273-282.

14. Wong WE, Qi Y, Zhao L, Cai K. Effective Fault Localization using Code Coverage. Proceedings of the 31st Annual IEEE Computer Software and Applications Conference 2007; :449-456.

15. Lee HJ. Software Debugging Using Program Spectra. PhD Thesis, University of Melbourne 2011.

16. Groce A, Chaki S, Kroening D, Strichman O. Error explanation with distance metrics. International Journal on Software Tools for Technology Transfer 2006; 8(3):229-247.

17. Gonzalez A. Automatic Error Detection Techniques based on Dynamic Invariants. Master's Thesis, Delft University of Technology, The Netherlands 2007.

18. Dallmeier V, Lindig C, Zeller A. Lightweight bug localization with ample. Proceedings of the sixth international symposium on Automated analysis-driven debugging, ACM, 2005; 99-104.

19. Hutchins M, Foster H, Goradia T, Ostrand T. Experiments of the effectiveness of dataflow-and controlflow-based test adequacy criteria. Proceedings of the 16th international conference on Software engineering, IEEE Computer Society Press, 1994; 191-200.

20. Vokolos FI, Frankl PG. Empirical evaluation of the textual differencing regression testing technique. Software Maintenance, 1998. Proceedings., International Conference On, IEEE, 1998; 44-53.

21. Do H, Elbaum SG, Rothermel G. Supporting controlled experimentation with testing techniques: An infrastructure and its potential impact. Empirical Software Engineering: An International Journal 2005; 10(4):405-435.

22. Walter D. Overfitting, regularization, and hyperparameters. https://dswalter.github.io/blog/ overfitting-regularization-hyperparameters/. Accessed: 2015-11-29.

23. Landsberg D, Chockler H, Kroening D, Lewis M. Evaluation of measures for statistical fault localisation and an optimising scheme. Fundamental Approaches to Software Engineering. Springer, 2015; 115-129.

24. Wilcoxon F. Individual comparisons by ranking methods. Biometrics bulletin 1945; 1(6):80-83.

25. Wong WE, Gao R, Li Y, Abreu R, Wotawa F. A survey on software fault localization 2009; .

26. Yu K, Lin M, Gao Q, Zhang H, Zhang X. Locating faults using multiple spectra-specific models. Proceedings of the 2011 ACM Symposium on Applied Computing, ACM, 2011; 1404-1410. 
27. Wen W. Software fault localization based on program slicing spectrum. Proceedings of the 34th International Conference on Software Engineering, IEEE Press, 2012; 1511-1514.

28. Lee HJ, Naish L, Ramamohanarao K. Effective software bug localization using spectral frequency weighting function. Computer Software and Applications Conference (COMPSAC), 2010 IEEE 34th Annual, 2010; 218-227, doi:10.1109/COMPSAC.2010.26.

29. Naish L. Probabilistic declarative debugging. Journal of Functional and Logic Programming July 2008; 2008(1).

30. Ochiai A. Zoogeographic studies on the soleoid fishes found in japan and its neighbouring regions. Bull. Jpn. Soc. Sci. Fish 1957; 22(9):526-530.

31. Naish L, Ramamohanarao K, et al.. Multiple bug spectral fault localization using genetic programming. Software Engineering Conference (ASWEC), 2015 24th Australasian, IEEE, 2015; 11-17.

32. Jones J, Bowring J, Harrold M. Debugging in parallel. Proceedings of the ISSTA 2007; :16-26.

33. Abreu R, Zoeteweij P, Van Gemund AJ. Spectrum-based multiple fault localization. Automated Software Engineering, 2009. ASE'09. 24th IEEE/ACM International Conference on, IEEE, 2009; 88-99.

34. Wong WE, Debroy V, Xu D. Towards better fault localization: A crosstab-based statistical approach. Systems, Man, and Cybernetics, Part C: Applications and Reviews, IEEE Transactions on 2012; 42(3):378-396.

35. Agrawal H, Horgan J, London S, Wong W. Fault localization using execution slices and dataflow tests. Software Reliability Engineering 1995; :143-151.

36. Agrawal H, DeMillo RA, Spafford EH. Debugging with dynamic slicing and backtracking. Software: Practice and Experience 1993; 23(6):589-616.

37. Hofer B, Wotawa F. Spectrum enhanced dynamic slicing for better fault localization. ECAI, 2012; 420-425.

38. Mayer W, Stumptner M. Model-based debugging-state of the art and future challenges. Electronic Notes in Theoretical Computer Science 2007; 174(4):61-82.

39. Mayer W, Stumptner M. Evaluating models for model-based debugging. Proceedings of the 2008 23rd IEEE/ACM International Conference on Automated Software Engineering, IEEE Computer Society, 2008; 128-137.

40. Wotawa F. Fault localization based on dynamic slicing and hitting-set computation. 2010 10th International Conference on Quality Software, IEEE, 2010; 161-170.

41. Wotawa F. On the relationship between model-based debugging and program slicing. Artificial Intelligence 2002; 135(1):125-143.

42. Mayer W, Abreu R, Stumptner M, van Gemund A. Prioritising model-based debugging diagnostic reports. Proceedings of the 19th International Workshop on Principles of Diagnosis, Citeseer, 2008.

43. Abreu R, Mayer W, Stumptner M, van Gemund AJ. Refining spectrum-based fault localization rankings. Proceedings of the 2009 ACM symposium on Applied Computing, ACM, 2009; 409-414.

44. Moon S, Kim Y, Kim M, Yoo S. Ask the mutants: Mutating faulty programs for fault localization. Software Testing, Verification and Validation (ICST), 2014 IEEE Seventh International Conference on, IEEE, 2014; 153-162.

45. Debroy V, Wong WE. Combining mutation and fault localization for automated program debugging. Journal of Systems and Software 2014; 90:45-60.

46. Papadakis M, Le Traon Y. Effective fault localization via mutation analysis: a selective mutation approach. Proceedings of the 29th Annual ACM Symposium on Applied Computing, ACM, 2014; 1293-1300.

47. Chekam TT, Papadakis M, Traon YL. Assessing and comparing mutation-based fault localization techniques. arXiv preprint arXiv:1607.05512 2016;

48. Papadakis M, Le Traon Y. Using mutants to locate" unknown" faults. Software Testing, Verification and Validation (ICST), 2012 IEEE Fifth International Conference on, IEEE, 2012; 691-700.

49. Papadakis M, Le Traon Y. Metallaxis-fl: mutation-based fault localization. Software Testing, Verification and Reliability 2015; 25(5-7):605-628.

50. Zeller A. Isolating cause-effect chains from computer programs. ACM SIGSOFT Software Engineering Notes 2002; 27(6):10.

51. Zeller A, Hildebrandt R. Simplifying and isolating failure-inducing input. Software Engineering, IEEE Transactions on $2002 ; 28(2): 183-200$.

52. Zhang X, Gupta N, Gupta R. Locating faults through automated predicate switching. Proceedings of the 28th international conference on Software engineering, ACM, 2006; 272-281.

53. Wong WE, Horgan JR, London S, Mathur AP. Effect of test set minimization on fault detection effectiveness. Proceedings of the 17th international conference on Software engineering, ACM, 1995; 41-50.

54. Hsu H, Jones J, Orso A. RAPID: Identifying bug signatures to support debugging activities. 23rd IEEE/ACM International Conference on Automated Software Enginering, 2008. ASE 2008, 2008; 439-442.

55. Briand LC, Labiche Y, Liu X. Using machine learning to support debugging with tarantula. Software Reliability, 2007. ISSRE'07. The 18th IEEE International Symposium on, IEEE, 2007; 137-146.

56. Le TDB, Oentaryo RJ, Lo D. Information retrieval and spectrum based bug localization: better together. Proceedings of the 2015 10th Joint Meeting on Foundations of Software Engineering, ACM, 2015; 579-590. 


\section{University Library}

\section{- M M N E R VA A gateway to Melbourne's research publications}

Minerva Access is the Institutional Repository of The University of Melbourne

Author/s:

Neelofar, N;Naish, L;Lee, J;Ramamohanarao, K

Title:

Improving spectral-based fault localization using static analysis

Date:

2017-01-01

Citation:

Neelofar, N., Naish, L., Lee, J. \& Ramamohanarao, K. (2017). Improving spectral-based fault localization using static analysis. SOFTWARE-PRACTICE \& EXPERIENCE, 47 (11), pp.1633-1655. https://doi.org/10.1002/spe.2490.

Persistent Link:

http://hdl.handle.net/11343/292288 